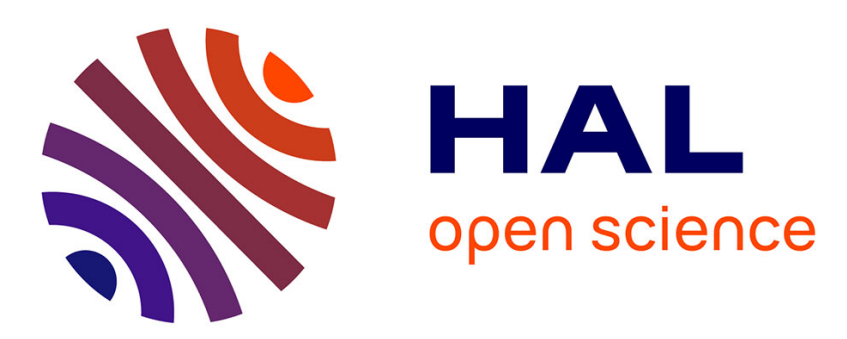

\title{
Utilisation d'un matériau à changement de phase dans un mur Trombe sans thermocirculation
}

\author{
L. Bourdeau
}

\section{To cite this version:}

L. Bourdeau. Utilisation d'un matériau à changement de phase dans un mur Trombe sans thermocirculation. Revue de Physique Appliquée, 1982, 17 (9), pp.633-642. 10.1051/rphysap:01982001709063300 . jpa-00245041

\section{HAL Id: jpa-00245041 https://hal.science/jpa-00245041}

Submitted on 1 Jan 1982

HAL is a multi-disciplinary open access archive for the deposit and dissemination of scientific research documents, whether they are published or not. The documents may come from teaching and research institutions in France or abroad, or from public or private research centers.
L'archive ouverte pluridisciplinaire HAL, est destinée au dépôt et à la diffusion de documents scientifiques de niveau recherche, publiés ou non, émanant des établissements d'enseignement et de recherche français ou étrangers, des laboratoires publics ou privés. 


\title{
Utilisation d'un matériau à changement de phase dans un mur Trombe sans thermocirculation (*)
}

\author{
L. Bourdeau $(* *)$ \\ Groupe d'Ecothermique Solaire du C.N.R.S. \\ Centre de Recherche Bâtiments Solaires CSTB/C.N.R.S. \\ BP 21, 06562 Valbonne cedex, France
}

(Reçule 21 septembre 1981, réviséle 8 février 1982, accepté le 15 mars 1982)

\begin{abstract}
Résumé. - Les murs Trombe sans thermocirculation (murs classiques de béton ou murs d'eau formés de réservoirs empilés ou cylindriques verticaux) sont maintenant très répandus dans l'habitat solaire passif, en particulier aux U.S.A. L'idée de réduire la taille de ces murs tout en améliorant leur comportement thermique s'est concrétisée avec la mise au point récente de matériaux à changement de phase fiables.

Des études théoriques et expérimentales faites dans une optique d'intégration de l'un d'entre eux, le chlorure de calcium hexahydraté, dans ce type de mur, conduisent à une meilleure appréhension du fonctionnement de cette paroi isotherme, et mettent en évidence un gain de $10 \%$ environ sur l'économie d'énergie réalisable et une réduction par un facteur 6 de la masse du stockage. Enfin, une étude de sensibilité montre comment varie cette économie avec la température de fusion et la chaleur latente massique du matériau.
\end{abstract}

\begin{abstract}
Unvented masonry or water Trombe walls have been widely applied to passive solar space heating. Recent development of reliable phase change materials (PCM) gives rise to the hope of reducing the mass and improving the thermal behaviour of these walls.

Calculations and experiments provide better understanding of the behaviour of such walls containing calcium chloride hexahydrate, and evidence a $10 \%$ higher Solar Savings Fraction when using them instead of masonry walls. Moreover the weight of PCM walls is one sixth that of masonry walls. An analysis of performance sensitivity to the phase change temperature and the latent heat of fusion will be found lastly.
\end{abstract}

1. Introduction. - L'étude de l'utilisation dans l'habitat solaire des matériaux à changement de phase (ou matériaux à chaleur latente) est maintenant sortie de sa phase prospective. En effet la disponibilité récente de divers matériaux $[1,2,3]$ a permis de mener à bien de nombreuses expériences en laboratoire ou en conditions climatiques réelles. Des applications réelles à l'habitat selon des configurations diverses ont pu alors être réalisées $[4,5,6]$.

L'intégration de ces matériaux dans des parois composites, en permettant d'obtenir une inertie importante dans un volume réduit et sous un faible intervalle de température, a fait naître de nouvelles solutions au

(*) Recherche effectuée avec l'aide du Los Alamos Scientific Laboratory, POB 1663, MS 571, Los Alamos, NM87545 sous les auspices de l'US Department of Energy, Office of Solar Applications.

$\left({ }^{* *}\right)$ Actuellement ingénieur au Département Energie Solaire du C.S.T.B. au Centre de Recherche Bâtiments Solaires à Valbonne. problème du chauffage solaire passif. La plus simple d'entre elles est celle d'un mur du type Trombe-Michel [7] dans lequel un matériau à changement de phase remplace les matériaux usuels de stockage (béton-eau) afin de créer une paroi isotherme. Nous rapportons ici les principaux résultats d'une étude effectuée sur ce mur dans le cas où le transfert des calories ne peut se faire que par conduction dans le mur c'est-à-dire pour une configuration sans thermocirculation (aussi appelée sans ventilation). Cette étude a été menée à la fois grâce à des expériences réalisées en plein hiver au Los Alamos Scientific Laboratory sur des murs construits dans des cellules test et grâce à des calculs effectués par simulation numérique sur ordinateur.

Après avoir brièvement rapporté au début du chapitre 2 les équations du modèle de simulation numérique utilisé dans nos calculs théoriques, nous décrivons les expériences qui ont été réalisées sur ce type de paroi sans ventilation et qui nous ont permis de valider le modèle et de dégager les caractéristiques du fonctionnement d'une telle paroi utilisant un matériau stabilisé 
à base de chlorure de calcium hexahydraté : la chliarolithe [1].

Ces données expérimentales ont été ensuite complétées par des études de sensibilité et des prédictions de performances annuelles obtenues par simulation numérique et rapportées aux chapitres 3 et 4 . Résultats expérimentaux et calculs permettent alors de dimensionner les parois isothermes et de chiffrer les avantages qu'apporte le remplacement du béton ou de l'eau par un produit comme la chliarolithe.

\section{Simulation numérique des parois isothermes. -} 2.1 InTRODUCTION. - Les simulations numériques par ordinateur des systèmes solaires, tant actifs que passifs, connaissent un développement très important. Le nombre des programmes disponibles (surtout aux U.S.A.) est énorme [8]. La raison est évidente.

Les expériences sur les systèmes solaires étant très longues et onéreuses, on cherche généralement à construire un modèle mathématique formé d'équations décrivant le mieux possible les phénomènes physiques intervenant dans le système. Des solutions analytiques n'existent que pour les systèmes très simples et pour des conditions aux limites irréalistes. On est donc très vite amené à considérer une résolution par ordinateur. Le modèle numérique obtenu est alors un outil de recherche très utile pour l'étude du système et en particulier son optimisation. Le comportement du modèle doit évidemment être comparé au comportement du système réel dans le plus grand nombre de conditions possibles. Cette méthode de travail est donc essentiellement inductive et doit toujours être utilisée avec précaution.

Divers programmes, comme Doe ou Blast aux U.S.A., utilisent la méthode des facteurs de réponse. Cette méthode ne peut traiter aisément les variations de configuration, comme celles apportées par une isolation amovible, ou les phénomènes non linéaires dus par exemple à la présence d'un matériau à changement de phase (problème de Stefan).

Une autre solution réside dans la méthode des différences finies. Cette méthode, de conception très simple, ne connaît en fait pratiquement pas de limitations et permet de traiter des configurations complexes avec des temps machine raisonnablement limités. Elle a été largement utilisée sous ses deux formulations par le Los Alamos Scientific Laboratory (formulation implicite) dans Pasole [9] et par l'Université de Californie à San Diego (formulation explicite) [10]. Ici, la méthode, sous sa formulation explicite, a été extrapolée afin de pouvoir traiter le cas des matériaux à changement de phase. Un sous programme, nommé CHALAT (chaleur latente), a alors été écrit afin de simuler sur ordinateur le comportement thermique d'un matériau à changement de phase intégré dans une paroi isotherme. A partir de ce sous-programme, il a enfin été conçu un programme nommé MURLAT (mur latent), permettant de décrire le fonctionnement d'un local unizone équipé d'une paroi isotherme.
MURLAT a été validé en deux temps :

a) Comparaison des simulations et des solutions analytiques dans un cas théorique simple.

b) Comparaison des simulations et des résultats expérimentaux obtenus sur des murs Trombe non ventilés soumis à des conditions climatiques réelles dans des modules test de quelques mètres cubes.

2.2 LA MÉTHODE DES DIFFÉRENCES FINIES APPLIQUÉES AUX MATÉRIAUX À CHANGEMENT DE PHASE. L'équation de la chaleur pour un matériau homogène inerte s'écrit, dans le cas d'un traitement unidimensionnel :

$$
\rho C_{\mathrm{p}} \frac{\partial T}{\partial t}=\lambda \frac{\partial^{2} T}{\partial x^{2}}
$$

où $T$ est la température, $t$ le temps et $\alpha=\lambda / \rho C_{\mathrm{p}}$ la diffusivité thermique. En présence de changement de phase mettant en jeu une chaleur latente de fusion $L_{\mathbf{F}}$ la vitesse $V$ de propagation de la zone de changement de phase est donnée par :

$$
L_{\mathrm{F}} \rho \frac{\partial V}{\partial x}=\lambda \frac{\partial^{2} T}{\partial x^{2}} .
$$

Ces équations peuvent être traitées numériquement en discrétisant les variables $x$ et $t$. Les variables $T$ ou $V$ sont alors constantes dans chaque segment $\Delta x$ pendant un intervalle de temps $\Delta t$. Ces valeurs, $T_{n}$ et $V_{n}$, sont associées au nœud $n$ situé au centre du segment $n$. Les différentielles $\delta T_{n}$ et $\delta^{2} T_{n}$ s'écrivent, en utilisant les différences centrales :

$$
\begin{aligned}
\delta T_{n}=T_{n+1 / 2}-T_{n-1 / 2} & \\
\delta^{2} T_{n}=\delta\left(\delta T_{n}\right)=\delta T_{n+1 / 2} & -\delta T_{n-1 / 2}= \\
& =T_{n+1}-2 T_{n}+T_{n-1} .
\end{aligned}
$$
La dérivée seconde $\frac{\partial^{2} T}{\partial x^{2}}$ peut alors être calculée par
l'approximation :

$$
\frac{\partial^{2} T_{n}}{\partial x^{2}}=\frac{\delta^{2} T_{n}}{\Delta x^{2}}=\frac{T_{n+1}-2 T_{n}+T_{n-1}}{\Delta x^{2}} .
$$

(1) devient

$$
\rho C_{\mathrm{p}} \Delta x \frac{\Delta T_{n}}{\Delta t}=\frac{\lambda}{\Delta x}\left(T_{n+1}-2 T_{n}+T_{n-1}\right)
$$

(2) devient

$$
\rho L_{\mathrm{F}} \Delta x \frac{\Delta Y_{n}}{\Delta t}=\frac{\lambda}{\Delta x}\left(T_{n+1}-2 T_{n}+T_{n-1}\right)
$$

où $Y_{n}$ est une variable sans dimension comprise entre 0 et 1. $Y_{n}$, taux de fusion du segment, est le rapport de la masse de matériau fondu sur la masse totale de matériau dans le segment considéré.

En intégrant (de $t$ à $t+\Delta t$ ) l'équation (1') écrite 
sous sa forme différentielle avec $\mathrm{d} T_{n} / \mathrm{d} t$ on obtient

avec

$$
\rho C_{\mathrm{p}} \Delta x\left(T_{n}^{+}-T_{n}^{0}\right)=\int_{t}^{t+\Delta t} \frac{\lambda}{\Delta x} S_{n} \mathrm{~d} t
$$

$$
\begin{aligned}
& T^{+}=T(t+\Delta t) \\
& T^{0}=T(t) \\
& S_{n}=T_{n+1}-2 T_{n}+T_{n-1} .
\end{aligned}
$$

La différence entre les formulations explicite et implicite de la méthode réside dans le choix de l'approximation de l'intégrale

$$
\int_{t}^{t+\Delta t} S_{n} \mathrm{~d} t
$$

Celle-ci peut s'écrire :

$$
\int_{t}^{t+\Delta t} S_{n} \mathrm{~d} t=\left(\mu S_{n}^{0}+(1-\mu) S_{n}^{+}\right) \Delta t
$$

- Si $\mu=1$ (3) devient

$$
\rho C_{\mathrm{p}} \Delta x\left(T_{n}^{+}-T_{n}^{0}\right)=\frac{\lambda}{\Delta x} S_{n}^{0} \Delta t
$$

et $T_{n}^{+}$se calcule explicitement. C'est la méthode des

$$
\begin{aligned}
& \rho L_{\mathrm{F}} \Delta x\left(Y_{n}^{+}-Y_{n}^{0}\right)=\frac{\lambda}{\Delta x} S_{n}^{0} \Delta t \\
& \rho L_{\mathrm{F}} \Delta x\left(Y_{n}^{+}-Y_{n}^{0}\right)=\frac{\lambda}{\Delta x} S_{n}^{+} \Delta t \\
& \rho L_{\mathrm{F}} \Delta x\left(Y_{n}^{+}-Y_{n}^{0}\right)=\frac{\lambda}{\Delta x} \frac{S_{n}^{+}+S_{n}^{0}}{2} \Delta t
\end{aligned}
$$

différences finies explicites encore appelée : méthode des différences « en avant » (forward differences).

- Si $\mu=0$ (3) devient

$$
\rho C_{\mathrm{p}} \Delta x\left(T_{n}^{+}-T_{n}^{0}\right)=\frac{\lambda}{\Delta x} S_{n}^{+} \Delta t
$$

et le calcul de $T_{n}^{+}$exige la résolution d'un système d'équations. C'est la méthode des différences finies implicites appelée méthode des différences « en arrière " (backward differences).

$$
\text { - Si } \mu=1 / 2 \text { (3) devient }
$$

$$
\rho C_{\mathrm{p}} \Delta x\left(T_{n}^{+}-T_{n}^{0}\right)=\frac{\lambda}{\Delta x} \frac{S_{n}^{+}+S_{n}^{0}}{2} \Delta t .
$$

C'est la méthode utilisée dans le programme de simulation de systèmes passifs PASOLE mis au point par le Los Alamos Scientific Laboratory (Los AlamosU.S.A.) [9].

Il s'agit encore d'une méthode implicite (appelée quelquefois méthode des différences centrales) nécessitant la résolution simultanée de plusieurs équations (de nombre égal à $N$, nombre de nouds de la discrétisation spatiale).

L'équation $\left(2^{\prime}\right)$ peut de même s'écrire suivant les trois formulations :
Les formulations (9) ou (10) utilisées conjointement avec (6) ou (7) conduisent d'abord à résoudre un système de $N-M$ équations à $N-M$ températures inconnues, $M$ étant le nombre de nouds où le phénomène de fusion (ou de solidification) est en cours. La température pour ces $M$ nouds est alors fixe et égale à la température de changement de phase $T_{\mathrm{F}}$ du matériau.

$M$ équations du type (9) ou (10) donnent ensuite par résolution immédiate l'évolution des $M$ taux de fusion. Ce processus est à renouveler à chaque pas de temps $\Delta t$.

De plus une "gestion des équations " est à effectuer à chaque pas par un ensemble de tests sur $T_{n}$ et $Y_{n}$ afin de déterminer dans quel état physique se trouve la masse de matériau associée à chaque nœud (solidedeux phases-liquide).

Un processus semblable doit être suivi lorsque l'on utilise les formulations (5) et (8), si ce n'est que chaque valeur $T_{n}^{+}$, ne dépendant que des températures $T^{0}$, s'obtient en ne résolvant plus qu'une seule équation.

Les schémas implicites permettent d'obtenir une plus grande précision dans les résultats, même au cours des périodes transitoires. Cependant le temps de calcul varie alors comme $N^{2}$ ou même $N^{3}$ dans le cas où l'inversion de la matrice du système d'équations doit être effectuée à chaque pas de temps par suite de modifications de configurations. Le temps de calcul du schéma explicite étant seulement proportionnel à $N$, est généralement plus faible, même si l'on utilise des discrétisations spatiales et temporelles très fines afin d'augmenter la précision des résultats.

Notons enfin que l'introduction de nouvelles équations permettant de traiter le cas des matériaux à changement de phase ne modifie pas les conditions habituelles nécessaires pour assurer la stabilité de la formulation explicite.

C'est cette formulation explicite qui a été utilisée dans les programmes CHALAT et MURLAT. 
2. 3 LE SOUS-PROGRAMmE CHALAT. - Le sousprogramme d'ordinateur CHALAT (chaleur latente) permet de simuler le fonctionnement d'une unité de stockage à chaleurs latente et sensible pour laquelle les transferts de chaleur ont lieu dans une seule direction. On suppose que l'unité est formée de deux parois renfermant le matériau à changement de phase. On distingue donc trois « murs » dans cette unité :

- mur extérieur

- mur à changement de phase

- mur intérieur

chacun de ces murs pouvant avoir des propriétés physiques différentes (conductivité thermique, masse volumique, chaleur spécifique, épaisseur). Cependant, seul le mur médian peut être sujet à un changement de phase ; il possède donc deux grandeurs caractéristiques supplémentaires : sa chaleur latente et sa température, supposée fixe, de changement de phase. Les hypothèses simplificatrices suivantes ont été utilisées :

- Les propriétés physiques du mur salin sont les mêmes dans les phases liquides et solides. L'épaississant incorporé dans la chliarolithe ayant pour effet de supprimer toute convection dans la phase liquide et la conductivité de la phase solide n'étant que légèrement supérieure à celle de la phase liquide, l'erreur introduite sur les conductivités est négligeable. La différence entre les chaleurs spécifiques est généralement plus élevée. Cependant la faible importance (10 à $15 \%$ ) du stockage sensible par rapport au stockage latent dans les conditions usuelles de température minimise l'erreur faite en considérant une chaleur spécifique moyenne commune pour les deux phases. Les variations de densité sont ignorées.

- Le matériau à changement de phase se comporte de manière idéale, c'est-à-dire que les phénomènes de surfusion, ségrégation, séparation de phases sont ignorés et que le changement de phase s'effectue à température constante.

2.4 LE PROGRAMME MURLAT. - Un programme de simulation nommé MURLAT (mur latent) a été bâti autour du sous-programme CHALAT afin de pouvoir effectuer des prédictions annuelles sur la réponse d'un local équipé d'un mur type Trombe sans thermocirculation. Cette réponse se traduit par une température unique associée à un nœud sans capacité thermique relié à l'extérieur et au mur solaire par des résistances thermiques. Les déperditions thermiques du local, à l'exclusion du mur solaire, sont rapportées à la surface du mur solaire, ce qui introduit la notion de charge thermique du mur (ou LCR pour Load Collector Ratio). Le programme permet de limiter les variations de la température du local entre une température maximale et une température minimale et de calculer les quantités d'énergie qu'il a fallu apporter (par un chauffage auxiliaire) ou rejeter (par ventilation) pour ne pas franchir ces limites. Le programme accumule également les temps de fonctionnement du chauffage auxiliaire et de la ventilation.
Les hypothèses simplificatrices suivantes ont d'autre part été adoptées :

- les apports internes ne sont pas envisagés

- les échanges radiatifs et convectifs sont décrits par des coefficients moyens constants.

Ce choix se justifie par le fait que le but recherché est de mener des études de sensibilité à certains paramètres et de comparer les performances de différentes parois solaires dans des conditions identiques et non de prédire avec exactitude ces performances dans une situation précise.

Il faut enfin définir deux grandeurs caractéristiques calculées par le programme :

- L'économie d'énergie réalisée (ou SSF pour Solar Savings Fraction) définie par SSF $=1-\frac{\text { AUXNT }}{\text { PNORT }}$

AUXNT étant la quantité d'énergie fournie par le chauffage auxiliaire et PNORT les pertes « normales ", égales aux pertes du local maintenu à la température de base $\left(18^{\circ} \mathrm{C}\right)$ et dans lequel la paroi solaire a été remplacée par une paroi adiabatique (approche du mur sud adiabatique du Los Alamos Scientific Laboratory).

- Le ratio de ventilation nécessaire, rapport de la quantité d'énergie qu'il a fallu rejeter sur les pertes normales.

Le sous-programme MURLAT utilise le FORTRAN IV (un passage sur CDC 7600 pour une simulation annuelle nécessite environ $5 \mathrm{~s}$ de temps $\mathrm{CPU}$ ). Une version moins souple (limitation du nombre de nœuds) a été écrite en BASIC et est utilisable sur ordinateur de burcalu.

\subsection{VALIDATION DE MURLAT PAR MODÉLISATION} D'UN SYSTÈME À SOLUTION ANALYTIQUE CONNUE. Une des solutions disponibles du problème de Stefan est la solution de Neumann. Cette solution s'applique au cas du corps unidimensionnel semi-infini dont la température est initialement égale à la température de fusion $T_{\mathrm{F}}$ du matériau et dont la surface libre est maintenue pour $t \geqslant 0$ à une température $T_{\mathrm{S}}$ provoquant un changement de phase. La température $T(x, t)$ est alors donnée par :

$$
\begin{aligned}
& \frac{T(x, t)-T_{\mathbf{F}}}{T_{\mathbf{S}}-T_{\mathbf{F}}}=1-\frac{\operatorname{erf}(x / 2 \sqrt{\alpha t})}{\operatorname{erf}(a / 2 \sqrt{\alpha})} \text { pour } 0 \leqslant x \leqslant X(t) \\
& T(x, t)=T_{\mathbf{F}} \quad \text { pour } \quad x \geqslant X(t)
\end{aligned}
$$

$a$ étant une constante donnée implicitement par la relation

$$
\frac{a}{2 \sqrt{\alpha}} \exp \left(\frac{a^{2}}{4 \alpha}\right) \operatorname{erf}\left(\frac{a}{2 \sqrt{\alpha}}\right)=\frac{C_{\mathrm{p}}\left|T_{\mathrm{F}}-T_{\mathrm{S}}\right|}{L_{\mathrm{F}} \sqrt{\pi}}
$$

et $X(t)$ la position du front de fusion par $X(t)=a \sqrt{t}$.

La précision obtenue lors de la simulation de ce problème par MURLAT a été étudiée. Il apparaît 
que des pas de temps de 15 à 30 min. et des segments d'environ 1 à $1,5 \mathrm{~cm}$ d'épaisseur donnent ici une bonne précision. En effet, l'erreur sur la position du front de fusion est alors trés inférieure au millimètre.

2.6 VALIDATION DE MURLAT GRÂCE À L'ÉTUDE EXPÉRIMENTALE DE MURS TYPE TROMBE SANS THERMOCIRCULATION. - Un mur formé de récipients parallélépipédiques en polyéthylène disposés sur des étagères en bois derrière un double vitrage a été expérimenté dans une cellule test de Los Alamos [11, 12]. Le mur contenait $500 \mathrm{~kg}$ de chliarolithe répartis dans 105 récipients de 31 environ. L'épaisseur moyenne de chliarolithe (épaisseur réelle des récipients multipliée par le pourcentage volumique d'occupation de la chliarolithe dans le mur) était voisine de $8 \mathrm{~cm}$. Un mur de conception identique mais plus petit $(21 \mathrm{~kg}$ de chliarolithe) a été étudié dans des boîtes-test. Des comparaisons avec le comportement de murs Trombe en béton et avec les résultats donnés par simulation grâce à MURLAT ont alors été effectuées. Il est apparu que :

- MURLAT permettait de reproduire fidèlement les températures enregistrées dans des modules test équipés de murs en béton ou à chliarolithe sur de longues séquences (Fig. 1).

- La température moyenne en mars dans le module contenant le mur à chliarolithe fut de $1,5^{\circ} \mathrm{C}$ plus élevée que dans le module contenant le mur de béton (Fig. 1). L'efficacité, rapport de l'énergie solaire utilisée à l'énergie solaire incidente, atteignit $36,7 \%$ contre $35 \%$ pour la période étudiée.

- La présence d'un matériau à changement de phase a conduit à une certaine stabilisation des températures dans le module à chliarolithe, lors de la période de charge du mur et surtout lors de la restitution (Fig. 2). Ainsi du 28 au 30 janvier, la température derrière le mur à chaleur latente a varié entre $22^{\circ} \mathrm{C}$ et $17^{\circ} \mathrm{C}$ seulement alors que pour les murs en béton

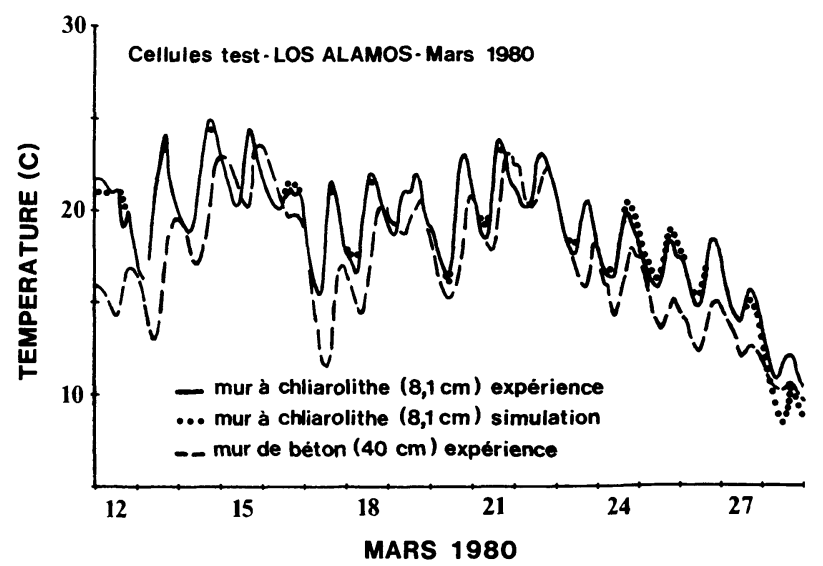

Fig. 1. - Températures du local derrière deux murs Trombe non ventilés (béton $40 \mathrm{~cm}$, chliarolithe $8,1 \mathrm{~cm}$ ).

[Room temperatures behind two unvented Trombe walls (40 cm, masonry wall and $8.1 \mathrm{~cm}$, PCM wall).]

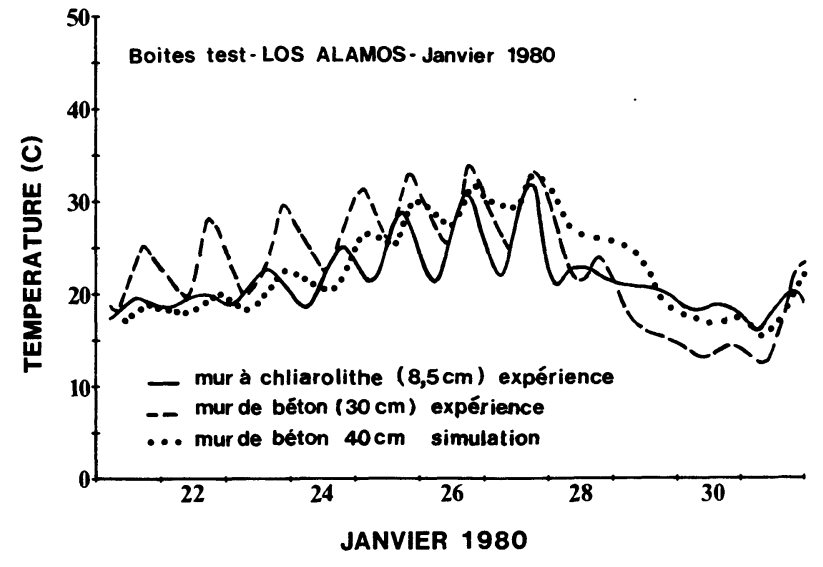

Fig. 2. - Températures du local derrière trois murs Trombe non ventilés (béton $40 \mathrm{~cm}$, béton $30 \mathrm{~cm}$, chliarolithe $8,5 \mathrm{~cm}$ ).

[Room temperatures behind three unvented Trombe walls $(40 \mathrm{~cm}$, masonry wall, $30 \mathrm{~cm}$, masonry wall and $8.5 \mathrm{~cm}$, PCM wall).]

on enregistra des chutes de $22^{\circ} \mathrm{C}$ à $11^{\circ} \mathrm{C}$ (mur de $30 \mathrm{~cm}$ ) et de $27^{\circ} \mathrm{C}$ à $16^{\circ} \mathrm{C}$ (mur de $40 \mathrm{~cm}$ ).

- Les variations journalières des températures (grandeur importante pour la notion de confort) derrière les murs de béton de grande épaisseur et derrière le mur à chliarolithe sont généralement très limitées (environ $3^{\circ} \mathrm{C}$ ). Toutefois une surchauffe $\mathrm{du}$ mur à chliarolithe apparaît lorsqu'après plusieurs journées très ensoleillées le mur ne peut plus accumuler d'énergie que par élévation de sa température. L'amplitude des oscillations de température peut alors atteindre une valeur double de celle des premiers jours (Fig. 2).

3. Etude théorique des performances d'un mur type Trombe sans thermocirculation. Comparaison de trois matériaux de stockage. - 3.1 INTRODUCTION. Le programme de simulation MURLAT a été utilisé pour mener une analyse de sensibilité à divers paramètres des performances d'un mur type Trombe sans ventilation. Rappelons que le modèle en question a été validé par comparaison avec des solutions analytiques connues et avec des résultats expérimentaux.

Il a d'autre part été précédemment utilisé afin d'analyser le comportement du mur sur des périodes courtes (un ou quelques jours) et sous des conditions quelque peu artificielles mais permettant de mettre en relief les caractéristiques de ce comportement en particulier en ce qui concerne les flux échangés [13].

Ici nous nous intéresserons aux performances globales mensuelles et annuelles du mur : économie d'énergie réalisée, ventilation et chauffage nécessaires au maintien du confort, rendement de captage et efficacité du mur.

Les données physiques utilisées sont les suivantes :

- béton : $C_{\mathrm{p}}=537 \mathrm{Wh} / \mathrm{m}^{3}{ }^{\circ} \mathrm{C} ; \lambda=1,65 \mathrm{~W} / \mathrm{m}{ }^{\circ} \mathrm{C}$ 
- chliarolithe :

$$
\begin{aligned}
& C_{\mathrm{p}}=845 \mathrm{Wh} / \mathrm{m}^{3}{ }^{\circ} \mathrm{C} ; \quad \lambda=0,7 \mathrm{~W} / \mathrm{m}^{\circ} \mathrm{C} \text {; } \\
& L_{\mathrm{F}}=63,3 \mathrm{kWh} / \mathrm{m}^{3} ; \quad T_{\mathrm{F}}=28,5^{\circ} \mathrm{C} \text {. }
\end{aligned}
$$

On suppose que le vitrage extérieur est double et que la température intérieure ne peut fluctuer qu'entre 18 et $22^{\circ} \mathrm{C}$.

Les données météorologiques utilisées sont les données horaires typiques des fichiers américains SOLMET-TMY. Dans ces fichiers, une année "typique» a été artificiellement construite pour chaque localité importante en assemblant les relevés météorologiques les plus représentatifs de chacun des douze mois. Les mois de l'année typique appartiennent donc généralement à des années réelles différentes.

Les résultats rapportés ci-dessous se réfèrent (sauf indication contraire), à des simulations de fonctionnement de murs situés à Albuquerque (Nouveau Mexique, U.S.A.). Les moyennes mensuelles, sur la période de novembre à mars, de l'ensoleillement par mètre carré vertical sud et des degrés-heures sont respectivement égales à $132 \mathrm{kWh}$ et 10130 degrésheures. Par comparaison, l'ensoleillement à Nice est $35 \%$ moins fort mais les degrés-heures y sont $40 \%$ plus faibles.

3.2 ECONOMIE D'ÉNERGIE RÉALISÉE. - La figure 3 rapporte les variations de SSF (économie d'énergie réalisée) en fonction de l'épaisseur du mur pour 3 matériaux et 3 valeurs de LCR (charge thermique du mur). On peut tirer plusieurs conclusions de ces graphes :

- Comme prévu, il existe une épaisseur optimale pour le mur de chliarolithe. Un stockage minimal est requis pour étaler sur toute la nuit la restitution des calories captées le jour. Si le mur est trop mince, il surchauffe le jour et tout le matériau de stockage gèle avant le lendemain matin. C'est pourquoi SSF augmente très vite avec l'épaisseur en dessous de ce minimum. Au-dessus de ce minimum, SSF augmente légèrement grâce à une capacité de stockage plus grande permettant d'étaler captage et restitution sur plus de $24 \mathrm{~h}$, puis décroît rapidement lorsque la résistance thermique globale du mur devient trop grande. Cette dernière remarque s'applique également au mur en béton dont la courbe présente également un maximum. Par contre, les performances du mur à eau ne sont pas limitées et augmentent indéfiniment (mais très lentement) avec la masse du stockage.

- L'économie maximale obtenue avec le mur à chliarolithe est dans tous les cas d'environ $10 \%$ plus forte que celle obtenue avec le mur à béton.

- Le mur à eau procure les économies les plus fortes mais nous verrons plus loin qu'il requiert une ventilation importante.

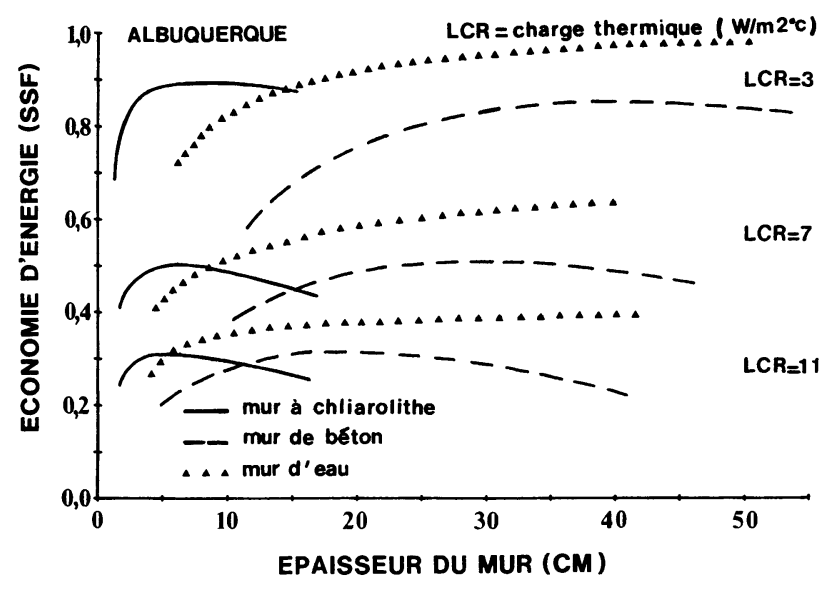

Fig. 3.

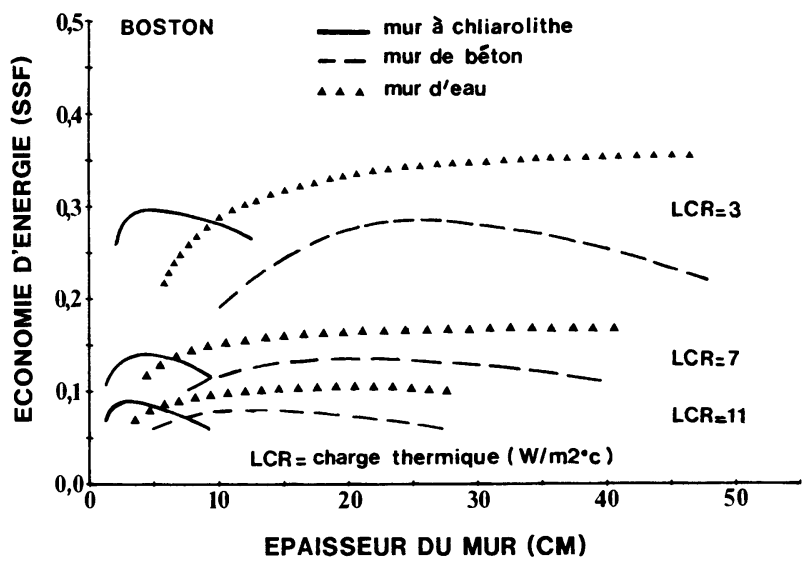

Fig. 4.

Figs. 3-4. - Economie d'énergie réalisée sur un hiver grâce à un mur Trombe non ventilé en fonction de l'épaisseur du mur, de la charge thermique du mur et du matériau de stockage.

[Effect of thermal storage thickness on the solar savings fraction obtained with an unvented Trombe wall for three storage materials and three values of the load collector ratio (LCR).]

- Les épaisseurs optimales du mur en chliarolithe sont égales au quart des épaisseurs optimales du mur en béton. On arrive même à un facteur 6 si l'on raisonne sur les masses. C'est sans doute là la différence la plus frappante entre les deux types de mur. Même en comparant avec le mur à eau, l'avantage de la chliarolithe est encore sensible. A même SSF on peut se contenter d'un volume deux à quatre fois moindre selon la charge thermique du mur.

On remarque que ces conclusions sont également valables pour un climat moins ensoleillé comme celui de Boston (Massachussets, U.S.A.) (Fig. 4).

3.3 VENTILATION NÉCESSAIRE AU CONTRÔLE DE LA TEMPÉRATURE MAXIMALE. - DeuX grandeurs caractérisant l'importance de cette ventilation ont été extraites de la simulation : d'une part, la quantité d'énergie qu'il a fallu rejeter, exprimée sur la figure 5 en pourcentage des pertes normales, d'autre part 


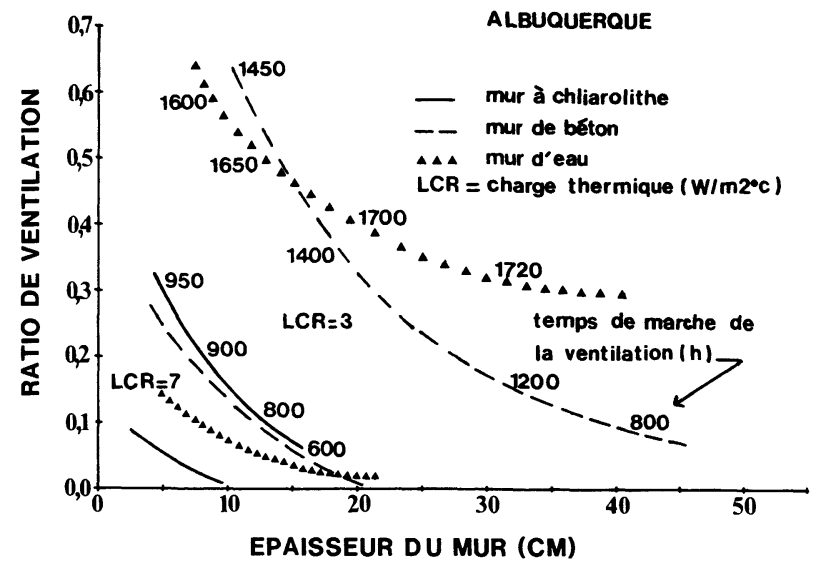

Fig. 5. - Ventilation nécessaire au contrôle de la température maximale dans un local derrière un mur Trombe non ventilé en fonction de l'épaisseur du mur, de la charge thermique du mur et du matériau de stockage.

[Effect of thermal storage thickness on the ventilation required to avoid overheating with an unvented Trombe wall for three storage materials and three values of the load collector ratio (LCR).]

la durée globale de fonctionnement de cette ventilation, indiquée en paramètre sur les courbes. On s'aperçoit que :

- La ventilation n'est importante que pour les faibles valeurs de LCR.

- Les ventilations nécessaires pour un mur de chliarolithe et un mur de béton d'épaisseurs optimales sont comparables (quoique légèrement plus fortes en quantité, mais non en durée pour la chliarolithe).

- Le mur à eau requiert des ventilations bien plus importantes que les deux autres types de mur pour les faibles valeurs de LCR.

Il faut remarquer que si une ventilation est nécessaire (mur à eau ou murs béton et chliarolithe de faible épaisseur), celle-ci requiert un système assez précis capable de ne rejeter que l'énergie excédentaire. Une simple ouverture de fenêtre par exemple, peut amener à une perte d'énergie beaucoup plus grande que nécessaire. C'est pourquoi, on cherchera plutôt à s'affranchir de ce besoin de ventilation. Ainsi l'épaisseur recommandée aux U.S.A. pour les murs Trombe en béton est-elle de 40 à $45 \mathrm{~cm}$ (cas des forts SSF) alors qu'une épaisseur de 20 à $25 \mathrm{~cm}$ fournirait sensiblement la même économie d'énergie. L'avantage $\mathrm{du}$ mur épais est que le système est entièrement passif. De même on préférera pour un mur en chliarolithe, une masse plus élevée ( 8 à $9 \mathrm{~cm}$ au lieu de 4 à $5 \mathrm{~cm}$ ).

3.4 RENDEMENT DE CAPTAGE. - Les courbes de rendement visibles sur la figure 6 ont la même forme que les courbes d'économie réalisée. Les épaisseurs optimales sont cependant ici plus petites, ce fait s'expliquant facilement en se rappelant qu'un mur

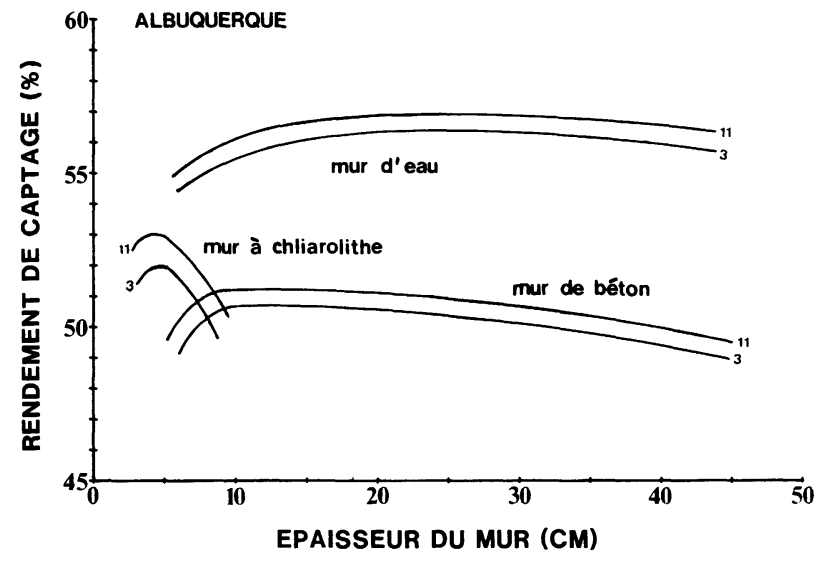

Fig. 6. - Rendement de captage d'un mur Trombe non ventilé en fonction de l'épaisseur du mur et du matériau de stockage (charge thermique du mur comprise entre 3 et $\left.11 \mathrm{~W} / \mathrm{m}^{2}{ }^{\circ} \mathrm{C}\right)$.

[Effect of thermal storage thickness on the collector efficiency of an unvented Trombe wall for three storage materials (the band shows the range of efficiency associated with a LCR of 3 up to $\left.11 \mathrm{~W} / \mathrm{m}^{2}{ }^{\circ} \mathrm{C}\right)$.]

mince nécessite la mise en route d'une ventilation (Fig. 5).

3.5 EFFICACITÉ DU MUR. - Les courbes de la figure 7 laissent voir que le pourcentage d'énergie solaire incidente réellement utilisée (efficacité du mur) est compris entre 15 et $35 \%$, les plus fortes valeurs étant bien sûr obtenues avec les fortes charges thermiques.

Cependant ces valeurs sont encore faibles en comparaison des rendements de captage obtenus. L'idée d'établir une isolation nocturne en liaison avec un stockage plus important vient à l'esprit comme solution pour obtenir des valeurs d'efficacité proches de celles du rendement. Des simulations effectuées par le Los Alamos Scientific Laboratory sur des murs Trombe en béton de $45 \mathrm{~cm}$ d'épaisseur, donnent par exemple les résultats suivants [11] :

$\underline{\mathrm{SSF}}\left\{\begin{array}{lllll}\text { LCR }\left(\mathrm{W} / \mathrm{m}^{2}{ }^{\circ} \mathrm{C}\right) & 3 & 7 & 11 & \\ \text { Sans isolation nocturne } & 0,73 & 0,42 & 0,32 & \text { double vitre } \\ \text { Avec } 2 \mathrm{~cm} \text { de polystyrène } & 0,86 & 0,56 & 0,43 & \text { simple vitre } \\ \text { Avec } 5 \mathrm{~cm} \text { de polystyrène } & 0,91 & 0,62 & 0,47 & \text { simple vitre }\end{array}\right.$

Or les pertes intervenant de nuit dans un mur à béton et dans un mur à chliarolithe sont pratiquement égales [13]. Il est donc normal d'obtenir aussi par l'usage d'une isolation devant un mur à chliarolithe, une augmentation très nette de l'économie d'énergie réalisée, comme le montrent les quelques simulations effectuées en incorporant une isolation nocturne. Ce résultat peut également être obtenu en séparant la face captante du volume de stockage par une isolation fixe, le transfert de chaleur se faisant par une boucle d'air en convection forcée. C'est le principe du mur diode dont on trouvera une étude en [13]. 


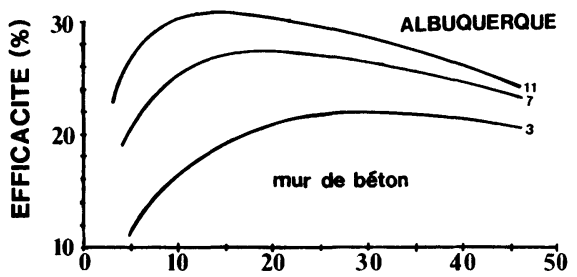

EPAISSEUR DU MUR (CM)
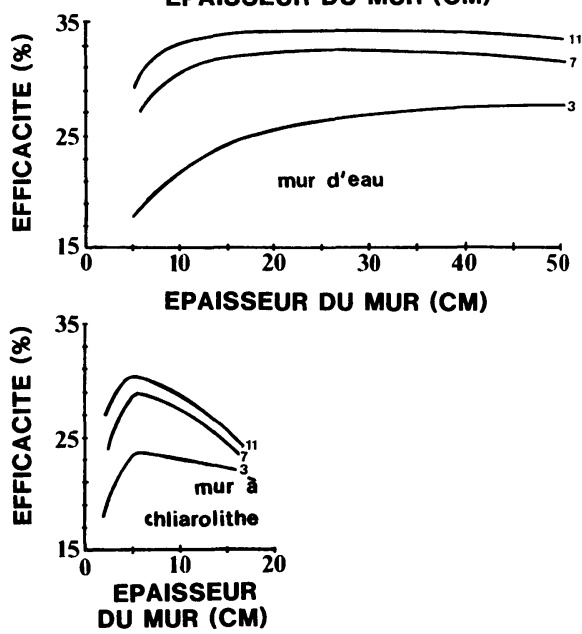

Fig. 7. - Efficacité d'un mur Trombe non ventilé en fonction de l'épaisseur du mur et du matériau de stockage (charge thermique du mur comprise entre 3 et $11 \mathrm{~W} / \mathrm{m}^{2}{ }^{\circ} \mathrm{C}$ ).

[Effect of thermal storage thickness on the solar heating system efficiency of an unvented Trombe wall for three storage materials (the band shows the range of efficiency associated with a LCR of 3 up to $11 \mathrm{~W} / \mathrm{m}^{2}{ }^{\circ} \mathrm{C}$ ).]

4. Etude théorique de la sensibilité des performances d'une paroi isotherme vis-à-vis de la température de fusion et de la chaleur latente massique du matériau à changement de phase . - - 4.1 SENSIBILITÉ À LA TEMPÉRATURE DE FUSION. - Considérons une paroi type Trombe sans thermocirculation et à chaleur latente. Quelques résultats théoriques et expérimentaux obtenus dans le cas où le matériau à changement de phase utilisé a une température de fusion de $28,5^{\circ} \mathrm{C}$ et une chaleur latente de $150000 \mathrm{~J} / \mathrm{kg}$ (cas de la chliarolithe) ont été rapportés au chapitre précédent. Il était évidemment intéressant d'étudier l'influence de la valeur de la température de fusion du matériau sur les performances du mur. Les résultats d'une simulation numérique menée sur une saison hivernale de novembre à mars sont donnés en figure 8 , sur laquelle a été portée en ordonnée l'économie d'énergie réalisée (Solar Savings Fraction) au cours de la période. Il apparaît qu'il existe une température optimale fonction du climat, de la charge du mur (coefficient de pertes thermiques de la maison par unité de surface du mur solaire) et du coefficient d'échanges thermiques entre le mur et l'espace habitable $h$.

Considérons, par exemple, la courbe relative à Albuquerque (climat très ensoleillé mais froid) et à une très faible charge thermique (habitat de $100 \mathrm{~m}^{2}$

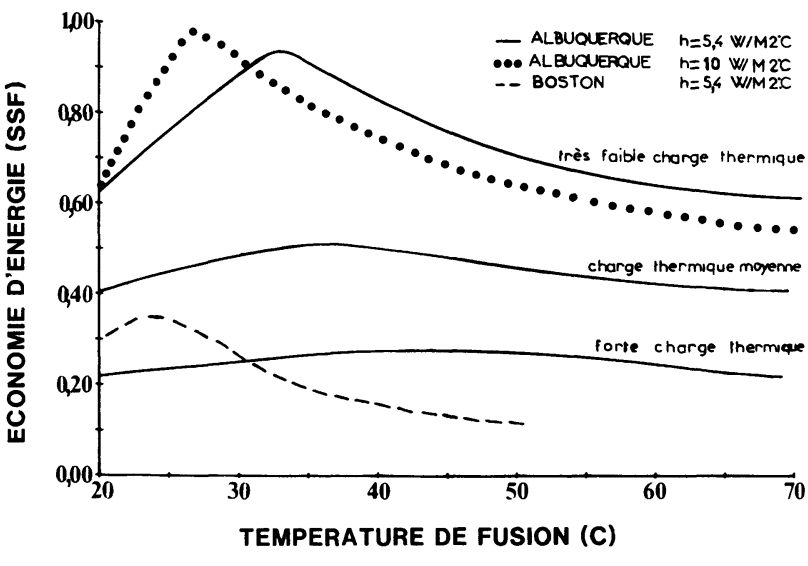

Fig. 8. - Economie d'énergie réalisée sur un hiver grâce à un mur Trombe non ventilé à chaleur latente en fonction de la température de fusion du matériau à changement de phase.

[Effect of PCM melting temperature on the solar savings fraction obtained with an unvented Trombe wall.]

équipé de $40 \mathrm{~m}^{2}$ de mur solaire et ayant un coefficient $G$, à l'exclusion du mur solaire, voisin de $\left.0,8 \mathrm{~W} / \mathrm{m}^{3} .{ }^{\circ} \mathrm{C}\right)$. L'optimum de la température de fusion est d'environ $31^{\circ} \mathrm{C}$. Il est possible d'expliquer pourquoi l'économie d'énergie réalisée décroît si la température de fusion s'éloigne de cette valeur en remarquant que la valeur des échanges thermiques à l'avant et à l'arrière du mur, excepté l'absorption du rayonnement solaire, dépend essentiellement de la valeur de la température de fusion du matériau $T_{\mathrm{F}}$.

a) Si $T_{\mathrm{F}}$ augmente, le rendement de captage du rayonnement solaire diminue par suite de l'élévation de la température de surface du mur; par ailleurs la puissance de la restitution d'énergie à la maison augmente et les périodes pendant lesquelles une ventilation de la maison s'avère nécessaire, par suite d'un trop fort apport d'énergie, s'en trouvent allongées. Il est donc normal que les performances annuelles du mur décroissent.

b) Si $T_{\mathrm{F}}$ diminue, le rendement de captage augmente mais le fait prépondérant est que la puissance de la restitution d'énergie à la maison diminue. L'énergie captée et stockée ne peut alors être restituée à la maison avec une puissance suffisante.

Il existe donc une température de fusion optimale. Cet optimum se déplace vers les hautes températures lorsque la charge thermique du mur augmente. En effet l'habitat a alors besoin d'un flux d'énergie en provenance du mur plus important afin de compenser des pertes thermiques vers l'extérieur plus fortes. De meilleurs échanges thermiques entre la surface du mur et l'habitat conduisent évidemment à une diminution de la température de fusion optimale. Cette dernière est également plus basse dans des conditions climatiques comme Boston pour lesquelles les longues périodes de fort ensoleillement sont très 
rares et par conséquent les risques de surchauffe plus faibles.

Notons que le fait d'avoir raisonné pour chaque $T_{\mathbf{F}}$ sur la valeur de SSF obtenue pour une épaisseur de matériau fixe de $6 \mathrm{~cm}$ (capacité d'accumulation de $4 \mathrm{kWh} / \mathrm{m}^{2}$ ), et non pour l'épaisseur optimale correspondante, se justifie en remarquant que cette valeur de SSF est toujours très proche de la valeur maximale.

Cette brève étude montre que la température de fusion optimale d'un matériau à changement de phase (de chaleur latente et conductivité thermique voisines de celles de la chliarolithe), inséré dans un mur Trombe non ventilé, est comprise entre 26 et $32^{\circ} \mathrm{C}$ lorsque l'on cherche à obtenir des économies d'énergies importantes. Cette conclusion peut logiquement s'appliquer à toute paroi chauffante (mur, dalle) dès lors que la surface de restitution est de valeur voisine de la surface de captage de l'énergie solaire.

4.2 Sensibilité À la ChaleUr latente MASSIQUE. - La température et la chaleur latente de fusion de la chliarolithe sont variables avec les quantités d'impuretés $(\mathrm{KCl}, \mathrm{NaCl}$ par exemple) qu'elle contient. Le point de fusion du chlorure de calcium hexahydraté pur est égal à $29,8^{\circ} \mathrm{C}$ et sa chaleur latente atteint alors la valeur de $170000 \mathrm{~J} / \mathrm{kg}$. Pratiquement, on obtient pour la chliarolithe des chaleurs latentes comprises entre 130000 et $150000 \mathrm{~J} / \mathrm{kg}$, cette dernière valeur correspondant au maximum de la valeur associée au produit pur mais corrigée de la présence de $12 \%$ en masse de terre de diatomites, produit inerte. A l'opposé, l'adjonction de fortes quantités de $\mathrm{SrCl}_{2}$ $6 \mathrm{H}_{2} \mathrm{O}$ (quelques pour cent) semble conduire à une élévation de la chaleur latente du produit. Il était donc intéressant d'étudier comment varie, avec la chaleur latente massique du matériau utilisé, l'économie d'énergie réalisée sur une saison hivernale de novembre à mars. La figure 9 rapporte les résultats d'une simulation numérique menée dans les mêmes conditions qu'au paragraphe 4.1 .

Trois courbes ont été tracées, correspondant chacune à une valeur de la chaleur latente massique du matériau stockeur $(100000,150000,200000 \mathrm{~J} / \mathrm{kg})$. La chaleur latente portée en abscisse est la chaleur latente totale du mur. Les caractéristiques thermiques du matériau, autres que sa chaleur latente massique, sont invariables et égales à celles du chlorure de calcium hexahydraté. Les différences dans les performances obtenues par des murs ayant une même chaleur latente totale sont donc causées par des résistances thermiques globales et des capacités de stockage sensible globales différentes. Il apparaît qu'il existe une valeur de la chaleur latente totale $\left(2 \mathrm{kWh} / \mathrm{m}^{2}\right)$ pour laquelle les économies d'énergie réalisées grâce aux trois murs sont pratiquement égales. Notons que cette valeur est d'ailleurs de l'ordre de grandeur de la valeur du stockage nécessaire à étaler sur $24 \mathrm{~h}$ la restitution de l'énergie captée le jour. Les positions relatives des

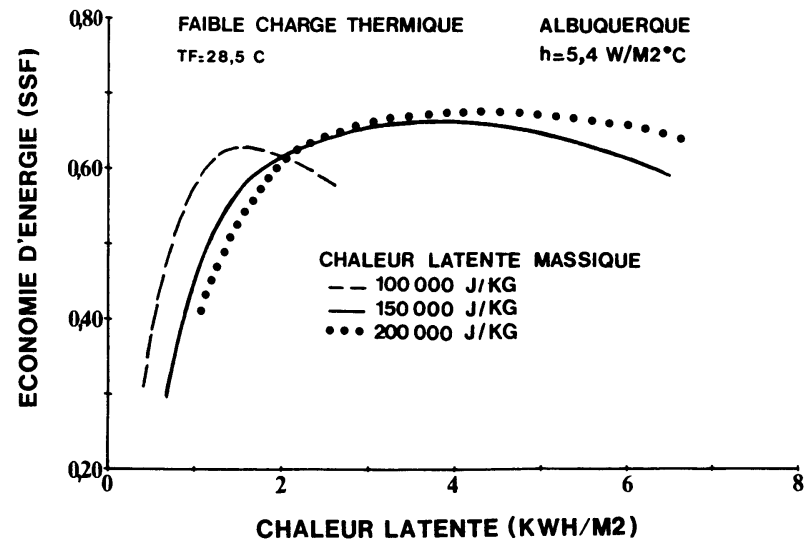

Fig. 9. - Economie d'énergie réalisée sur un hiver grâce à un mur Trombe non ventilé à chaleur latente en fonction de la chaleur latente totale par $\mathrm{m}^{2} \mathrm{du}$ mur et de la chaleur latente massique du matériau à changement de phase.

[Effect of thermal storage capacity on the solar savings fraction obtained with an unvented PCM Trombe wall for three values of the latent heat of fusion.]

trois courbes en deçà et au-delà de cette valeur s'expliquent facilement en considérant les épaisseurs des murs : en deçà, le mur contenant un matériau à faible chaleur latente massique est favorisé par son importante capacité de stockage par chaleur sensible, au-delà, sa grande résistance thermique globale le pénalise.

Mais le point important à constater est que les faibles variations de chaleur latente massique du matériau conduisent à de très faibles variations des performances du mur. Ainsi une augmentation de 150000 à $200000 \mathrm{~J} / \mathrm{kg}$ de la chaleur latente massique est certes favorable du point de vue du dimensionnement du mur, mais non du point de vue de ses performances thermiques.

4.3 ConClusion. - La chliarolithe est apparue comme étant un matériau adéquat pour les applications, tout au moins passives, de l'énergie solaire à l'habitat. Ce matériau dispose en effet, compte tenu de ses autres propriétés thermiques, d'une température de fusion très proche des températures optimales nécessaires à l'obtention de performances élevées. De plus, il n'est nullement pénalisé, d'un point de vue thermique, par une chaleur latente qui aurait pu paraître faible par rapport à celle d'autres matériaux.

5. Conclusion. - Le mur Trombe, à eau ou en béton, est sans doute un des systèmes de chauffage solaire passif les mieux connus et les plus répandus, en particulier aux USA dans des régions de climat froid et ensoleillé. Son utilisation sous sa forme originale met en jeu une thermocirculation diurne entre la pièce à chauffer et la face avant du mur, ce qui permet d'abaisser la température de surface du capteur et d'augmenter les rendements de captage. Malheureusement pour les systèmes largement dimensionnés, 
cet apport de calories conduit à une surchauffe de la pièce. On peut certes, prévoir une ventilation forcée et un stockage auxiliaire chargés d'emmagasiner ces calories. Cette solution non passive, est plus complexe. C'est pourquoi un grand nombre des murs Trombe actuellement en fonctionnement, sont du type sans ventilation, solution simple et procurant un certain confort [11]. L'installation d'une isolation extérieure amovible apporte un gain considérable mais est encore peu courante (problèmes d'esthétique, de fragilité, de servitude, voire de prix, si le système est automatisé).

Mais le gros inconvénient du mur Trombe (à eau ou en béton) réside dans sa masse élevée. Le remplacement de cette masse de stockage à chaleur sensible par un matériau de stockage à chaleur latente comme d'un facteur 4 et sa masse d'un facteur 6 (cas du béton). De plus, le fonctionnement thermique du mur s'en trouve amélioré. Grâce à des rendements de captage légèrement plus élevés et à une restitution plus régulière des calories, l'économie d'énergie réalisée augmente d'environ $10 \%$. Enfin dans le cas où la température de la pièce n'est pas artificiellement contrôlée, on obtient avec la chliarolithe une meilleure stabilisation de cette température.

Remerciements. - L'auteur exprime ici ses remerciements à Doug Balcomb et à tous les membres du Los Alamos Scientific Laboratory pour l'aide apportée à la réalisation de cette étude.

\section{Bibliographie}

[1] Schneider, M., Sylvain, J. D., Berger, X., Jaffrin, A., Bourdeau, L., Matériau accumulateur de calories à température constante et applications de ce matériau. Brevet français no $79-13296$, Brevet européen no 80 430010-1.

[2] Telkes, M., Sel de Glauber stabilisé. US Patent 3986969 de 1976.

[3] Capitant, M., Chabanon, F., Lenotre, C., Michel, E., Royer, H., Stockage de calories à bas niveau dans un sel fondu. ENSMP, 1978.

[4] Bourdeau, L., Etude de l'intégration à l'habitat d'éléments de parois isothermes. Thèse de DocteurIngénieur ECP, décembre 1980.

[5] Henry, J. J., Conception et expérimentation d'une maison solaire avec stockage par matériau à chaleur latente. Mémoire CNAM, Aix-en-Provence, octobre 1979.

[6] Clément, R., Berger, X., La maison solaire de Mougins. Colloque Solaire International. Nice, décembre 1980.

[7] Trombe, F., Maisons solaires. Techniques de l'ingénieur (3) 1974 C.
[8] SERI, Analysis Methods for Solar Heating and Cooling Applications. January 1980. SERI/SP-35-232 R. Solar Energy Research Institute.

[9] McFarland, R. D., Pasole, a general Simulation Program for Passive Solar Energy. October 1978. Informal Report LA-7433-MS Los Alamos Scientific Laboratory.

[10] Doe Final RePort : Impact of Controls in Passive Solar Heating and Cooling of Buildings. DE ACO4-79AL10891.

[11] Los Alamos Scientific Laboratory : Passive Solar Design Analysis DOE/CS-0127/2.

[12] Bourdeau, L., Study of two Passive Solar Systems Containing Phase-change Materials for Thermal Storage. 5th Passive Conference Amherst, Massachussets, october 1980. LA-UR-80-1669 Report.

[13] Bourdeau, L., Jafrrin, A., Moisan, A., Captage et stockage d'énergie solaire dans l'habitat par le moyen de mur diode à chaleur latente. Revue Phys. Appl. 15 (1980) 559-568. 